7-1-2019

\title{
When an Infected Meniscus Portends a Perforated Viscus
}

Michelle Hannon

Thomas Jefferson University

Stephen Zachariah

Thomas Jefferson University

Neha Bansal Etherington

Thomas Jefferson University

Anusha Govind

Thomas Jefferson University

Devin Weber

Thomas Jefferson University

Follow this and additional works at: https://jdc.jefferson.edu/didem

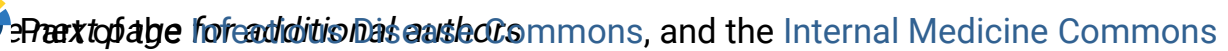
Let us know how access to this document benefits you

\section{Recommended Citation}

Hannon, Michelle; Zachariah, Stephen; Etherington, Neha Bansal; Govind, Anusha; Weber, Devin; and Hess, Bryan, "When an Infected Meniscus Portends a Perforated Viscus" (2019). Division of Infectious Diseases and Environmental Medicine Faculty Papers. Paper 7.

https://jdc.jefferson.edu/didem/7

This Article is brought to you for free and open access by the Jefferson Digital Commons. The Jefferson Digital Commons is a service of Thomas Jefferson University's Center for Teaching and Learning (CTL). The Commons is a showcase for Jefferson books and journals, peer-reviewed scholarly publications, unique historical collections from the University archives, and teaching tools. The Jefferson Digital Commons allows researchers and interested readers anywhere in the world to learn about and keep up to date with Jefferson scholarship. This article has been accepted for inclusion in Division of Infectious Diseases and Environmental Medicine Faculty Papers by an authorized administrator of the Jefferson Digital Commons. For more information, please contact: JeffersonDigitalCommons@jefferson.edu. 
Authors

Michelle Hannon, Stephen Zachariah, Neha Bansal Etherington, Anusha Govind, Devin Weber, and Bryan Hess 
Title: When an Infected Meniscus Portends a Perforated Viscus

\section{Authors:}

Michelle Hannon, $\mathrm{MD}^{1}$

Stephen Zachariah, $\mathrm{MD}^{1}$

Neha Bansal Etherington $\mathrm{MD}^{1}$

Anusha Govind $\mathrm{MD}^{2}$

Devin Weber $\mathrm{MD}^{2}$

Bryan Hess, $\mathrm{MD}^{2}$

\section{Affiliations:}

${ }^{1}$ Department of Medicine, Sidney Kimmel Medical College at Thomas Jefferson University, Philadelphia, Pennsylvania, United States.

${ }^{2}$ Department of Medicine, Division of Infectious Diseases, Sidney Kimmel Medical College at Thomas Jefferson University, Philadelphia, Pennsylvania, United States.

\section{Corresponding Author:}

Devin Weber, MD

Sidney Kimmel Medical College at Thomas Jefferson University

Division of Infectious Diseases

1015 Chestnut Street Suite 1020

Philadelphia, PA 19107

Telephone: 215-955-7785

Fax: 215-955-9362

Email: Devin.Weber@jefferson.edu

\section{Alternate Author:}

Bryan Hess, MD

Sidney Kimmel Medical College at Thomas Jefferson University

Division of Infectious Diseases

1015 Chestnut Street Suite 1020

Philadelphia, PA 19107

Telephone: 215-955-7785

Fax: 215-955-9362

Email: Bryan.Hess@ jefferson.edu 


\section{Acknowledgements:}

The authors would like to acknowledge Mindy Tokarczyk, with the Department of Clinical Laboratories at Thomas Jefferson University Hospitals and Brian O'Hara, MD with the Department of Clinical Pathology, for their contributions of clinical images to this case report.

\section{Funding:}

The authors have no source of funding to disclose.

\section{Conflicts of Interest:}

The authors have no conflicts of interest to disclose. 


\begin{abstract}
:
A 59-year-old woman status post renal transplant 7 years prior for autosomal dominant polycystic kidney disease on tacrolimus and mycophenolate mofetil presented with subacute left knee and right wrist pain. She received local steroid injections to both areas as an outpatient without improvement in her symptoms. She had recently traveled to India, her home country, to visit relatives. Aspiration of the knee revealed 4+ acid fast bacilli on smear and she was taken for surgical debridement. She was started on empiric antibiotics for presumed infection with rapidly growing Mycobacteria. Her course was complicated by acute onset abdominal pain with pneumoperitoneum and mesenteric abscess on imaging, requiring exploratory laparotomy. Surgical cultures from all sites were positive for Mycobacterium tuberculosis. Her medications were adjusted to rifampin, isoniazid, pyrazinamide, and ethambutol. Pre-transplant screening results were unknown. She was discharged in stable condition and completed 12 months of medical therapy.
\end{abstract}

Keywords: Mycobacterium tuberculosis; disseminated tuberculosis, GITB 


\section{Case Presentation:}

The patient was a 59-year-old woman with a history of a cadaveric renal transplant in 2011 for autosomal dominant polycystic kidney disease, immunosuppressed on tacrolimus and mycophenolate mofetil. She also reported a history of recurrent breast abscess of unknown etiology. She presented to our quaternary care center with progressive pain and swelling of the left knee and right wrist. Her symptoms initially started three months prior to admission with a mechanical fall resulting in left knee pain. She was evaluated by an orthopedic surgeon, who diagnosed her with a meniscal tear and gave her a local steroid injection. She then traveled to Kerala, India for two weeks. Upon her return, she started to note right wrist pain and swelling and again received a steroid injection in her right wrist. At this point, her symptoms worsened to the point that she reported to the emergency department. Accompanying symptoms included intermittent fevers, right breast drainage, and a 15-pound unintentional weight loss over these preceding three months.

Upon admission, ultrasound of the right wrist showed an approximately $2 \times 2 \mathrm{~cm}$ hypoechoic lesion along the dorsal aspect of the wrist. She was started on empiric vancomycin and cefepime for possible septic arthritis. An aspiration of the wrist at the bedside was attempted but only a small amount of fluid was extracted. An aspiration of her left knee effusion was notable for $25 \mathrm{~mL}$ of cloudy yellow fluid with a white blood cell count of 47,000 (60\% polymorphonuclear cells), no crystals, negative Gram stain, and 4+ acid fast bacilli (AFB) on AFB smear. There was initial concern for rapidly growing mycobacterial infection related to her corticosteroid injection. Subsequently, she was empirically started on oral clarithromycin, oral moxifloxacin, and intravenous imipenem/cilastatin. 
On day 4 of admission, she reported severe right upper quadrant abdominal pain. A computed tomography (CT) scan of the abdomen and pelvis revealed a large amount of peritoneal free air as well as a rim-enhancing, septated mass measuring 6.7 x 6.4 x $5.0 \mathrm{~cm}$. She was taken emergently to the operating room (OR) for an exploratory laparotomy, where she was found to have a perforation of the second part of her duodenum and a mesenteric abscess. AFB smear of the peritoneal fluid was negative and a routine OR culture grew only Candida albicans. The patient was transferred to the surgical intensive care unit (SICU) after this procedure, where hand surgery performed a washout of her wrist at the bedside.

On day 5 of admission, her knee synovial fluid cultures showed growth in the Mycobacterial Growth Indicator Tube (MIGT) and tested positive for Mycobacterium tuberculosis (MTB) by DNA probe. Cultures from her peritoneal fluid and right wrist tissue subsequently grew MTB [Figure 1]. Pathology from the knee synovial biopsy showed caseating synovium. Pathology from the duodenum and right wrist were also positive for AFB on histologic stain with granulomas [Figure 2]. She was transitioned to targeted MTB therapy with rifabutin, isoniazid, pyrazinamide, and ethambutol. Mycophenolate mofetil was discontinued to decrease her level of immunosuppression, but tacrolimus was continued to prevent allograft rejection.

The patient also reported a history of recurrent breast abscess, which had been debrided twice in the years prior to this hospitalization. We confirmed that routine cultures were negative previously. This area was debrided at the same time as her wrist joint at the bedside in the SICU, with no growth on routine cultures. Unfortunately, no AFB cultures or pathology samples were sent from this area. 
Our patient never had any respiratory complaints, though two of three sputum specimens were AFB positive. There was no evidence pulmonary tuberculosis on imaging. Blood cultures drawn throughout the hospitalization were negative for AFB and other pathogens. Though the etiology of her breast abscess remains unknown, she did not have a recurrence while on anti-tubercular therapy. At the time of this case report, the patient required 3 separate admissions for repeat debridement of the right wrist for osteomyelitis, with additional positive AFB stain but negative cultures. Therapy was extended in light of this and she has since completed 12 months of drug therapy in conjunction with the Department of Health.

\section{Discussion}

Reactivation of Mycobacterium tuberculosis is responsible for roughly 1.5 million deaths annually- the most by any single pathogen worldwide. In addition to these fatalities, nearly $25 \%$ of the global population is estimated to have latent tuberculosis (TB) infection $[1,2]$. India, Indonesia, China, the Philippines, and Pakistan have the largest numbers of new cases [2]. Immigrants from these countries as well as patients with risk factors such as alcohol and substance abuse disorders, homelessness, and incarceration largely contribute to the new cases of TB in the United States with 9,272 reported in 2016 [3,4]. Though pulmonary infection is most common, about $50 \%$ of immunocompromised patients experience extrapulmonary manifestations of TB compared to $20 \%$ of immunocompetent patients [5].

One rare extrapulmonary presentation of $\mathrm{TB}$ is gastrointestinal tuberculosis (GITB). It is commonly mistaken for Crohn's disease due to its propensity to cause scattered granulomatous lesions and nonspecific symptoms with a famous "mimicking" presentation. GITB most 
commonly affects the ileocecal area and right colon [5,6]. Involvement of the duodenum, as in our case, is extremely rare. Only about $2 \%$ of cases of GITB present in this way. Spread of MTB to the GI tract can be hematogenous, lymphatic, direct through the peritoneum, or via ingestion of contaminated substances [6]. Patients with GITB usually present with GI bleeding, fever, and abdominal pain, but may also have perforations, abscesses, and acute abdomen [5-8]. About 50\% of patients with GITB have disease isolated to the GI tract, whereas the other 50\% present with pulmonary or otherwise disseminated disease [7].

Osteoarticular TB represents another uncommon site of extrapulmonary TB, occurring in about $10 \%$ of extrapulmonary TB disease cases [10]. Based on two studies from Denmark and China including over 400 patients with bone and joint tuberculosis, 50 to $70 \%$ of cases involved the spine with the classic Pott's Disease; the knee represented 13-20\% of non-spine cases. About $80 \%$ of patients with any type of osteoarticular tuberculosis had a normal serum white blood cell count, but most had elevated inflammatory markers such as erythrocyte sedimentation rate and C-reactive protein as well as localizing symptoms such as pain and swelling overlying the affected joint. There is no reliable data describing the typical results of joint aspirates or histopathology. Only one patient studied had a positive AFB smear from joint aspirate and the only reliable method of disease confirmation was AFB culture. Both studies point out the insidious nature of osteoarticular $\mathrm{TB}$ and the long period of time between symptom onset and diagnosis [10,11]. Immunosuppression and recent joint injury may predispose patients to articular TB infections. Notably, only about $15 \%$ of patients with osteoarticular TB were found to have concomitant pulmonary involvement $[10,13]$. 
Another very rare, but notable presentation of $\mathrm{TB}$, is that of a mass mimicking a breast cancer; this has been reported several times in the literature $[12,14]$. Mammary TB is particularly unusual due to the highly aerobic nature of MTB [15]. It presents most commonly as a central or upper outer quadrant lump (70\% of cases), with most of the remainder presenting as ulcerations. Pain and discharge may be present, but are much less common. Mammary TB has frequently been mistaken for carcinoma or non-tubercular abscess. The spread of disease from lungs to the breast is hypothesized to occur via retrograde lymphatic flow, as axillary lymph nodes have been involved in up to $75 \%$ of mammary TB cases. Fine needle aspiration cytology is usually used to make the diagnosis, but core needle and open biopsies have also been used to confirm the presence of caseating granulomas $[16,17]$.

The Centers for Disease Control and Prevention (CDC) recommends that medical treatment for active tuberculosis be administered in two phases: a two-month intensive phase with isoniazid, rifampin, pyrazinamide, and ethambutol followed by a four- to seven-month continuation phase with isoniazid and rifampin. The length of the continuation phase is largely dependent upon whether or not cultures are found to be positive after the intensive phase $[18,19]$. When joint involvement is present, debridement is recommended in addition to the standard antitubercular pharmacotherapy with consideration of extending the continuation phase to 12 months [20]. There is no firm consensus on whether to manage uncomplicated GITB medically or surgically, but most studies suggest that resection followed by regular medical therapy is best to prevent complications such as perforation, tissue organization, and obstruction [21]. For mammary TB, standard antitubercular medical therapy has been used for treatment with a 95\% success rate. Surgical intervention is typically reserved for lack of resolution after medical therapy [17]. 
We report a case of an immunosuppressed patient with disseminated MTB infection who required combination medical and surgical management for successful outcome. This case highlights the importance of considering tuberculosis on the differential for patients in the United States who present with systemic infection in the setting of immunosuppression. It also calls to attention the need for appropriate pre-transplant screening and consultation with an Infectious Diseases expert to reduce morbidity and mortality from reactivated tuberculosis in the post-transplant period. 


\section{References}

1. Houben RM, Dodd PJ. The global burden of latent tuberculosis infection: A re-estimation using mathematical modelling. PLoS medicine. 2016;13(10):e1002152.

2. World Health Organization. Global tuberculosis report 2017. In: Global tuberculosis report $2017 ; 2017$.

3. Griffith DE, Kerr CM. Tuberculosis: Disease of the past, disease of the present. J Perianesth Nurs. 1996;11(4):240-245. doi: S1089-9472(96)80023-2 [pii].

4. Centers for disease control and prevention tuberculosis data and statistics. . Updated 2018. Accessed Feb, 2018.

5. Philpott H, Boussioutas A, Kronborg I, Zanatti S, Dow C. Gastrointestinal: Tuberculosis of the sigmoid colon—a cautionary tale. J Gastroenterol Hepatol. 2011;26(11):1692-1692.

6. Debi U, Ravisankar V, Prasad KK, Sinha SK, Sharma AK. Abdominal tuberculosis of the gastrointestinal tract: Revisited. World J Gastroenterol. 2014;20(40):14831-14840. doi: 10.3748/wjg.v20.i40.14831 [doi].

7. Jarrett O, Grim S, Benedetti E, Clark N. Gastrointestinal tuberculosis in renal transplant recipients: Case report and review of the literature. Transplant Infectious Disease. 2011;13(1):52-57.

8. Ngonge AL, Ekaney DSM, Sama C, Musonge-Effoe J, Effoe VS, Ekwen G. Multiple jejunal perforation secondary to intestinal tuberculosis infection: A case report. The Pan African medical journal. 2017;27.

9. Beppu K, Osada T, Matsumoto K, et al. Gastrointestinal tuberculosis as a cause of massive bleeding. Med Sci Monit. 2009;15(10):CS151-154. doi: 878200 [pii].

10. Johansen IS, Nielsen SL, Hove M, et al. Characteristics and clinical outcome of bone and joint 
tuberculosis from 1994 to 2011: A retrospective register-based study in denmark. Clinical Infectious Diseases. 2015;61(4):554-562.

11. Chen S, Zhao L, Dong W, et al. The clinical features and bacteriological characterizations of bone and joint tuberculosis in china. Scientific reports. 2015;5:11084.

12. Pattamapaspong N, Muttarak M, Sivasomboon C. Tuberculosis arthritis and tenosynovitis. . 2011;15(05):459-469.

13. Yun J, Sung H, Kim T, et al. Comparison of the clinical characteristics of mycobacterium tuberculosis and nontuberculous mycobacteria patients with joint involvement. Infection. 2015;43(2):207-209.

14. Teo T, Ho G, Chaturverdi A, Khoo B. Tuberculosis of the chest wall: Unusual presentation as a breast lump. Singapore Med J. 2009;50(3):e97-99.

15. Tanrikulu AC, Abakay A, Abakay O, Kapan M. Breast tuberculosis in southeast turkey: Report of 27 cases. Breast Care (Basel). 2010;5(3):154-157. doi: 000314267 [doi].

16. Thimmappa D, Mallikarjuna M, Vijayakumar A. Breast tuberculosis. Indian J Surg. 2015;77(3):1378-1384.

17. Mehta G, Mittal A, Verma S. Breast tuberculosis-clinical spectrum and management. Indian J Surg. 2010;72(6):433-437.

18. Centers for disease control and Prevention Treatment for TB disease. Updated 2016. Accessed Feb, 2018.

19. Nahid P, Dorman S, Alipanah N et al. Official American Thoracic Society/ Centers for Disease Control and Prevention/ Infectious Diseases Society of America Clinical Practice Guideline: Treatment of Drug- Susceptible Tuberculosis. Clin Infect Dis. 2016;63(7):147-195.

20. Veloci S, Mencarini J, Lagi F, et al. Tubercular prosthetic joint infection: Two case reports and 
literature review. Infection. 2017:1-14.

21. Pathak P, Sahu SK, Agrawal S, -. Clinico-pathological profile and surgical outcome of patients of gastrointestinal tuberculosis undergoing laparotomy. Chirurgia (Bucur). 2016;111(6):487492. doi: 10.21614/chirurgia.111.6.487 [doi]. 


\section{Figures}

Figure 1. Kinyoun Stain with serpentine cording from left knee synovium.

Figure 2. Caseating granulomas seen on pathology slide from right wrist tissue. 